\section{Caponi S. Uma sala tranquila. Neurolépticos para una biopolítica da indiferença. São Paulo: LiberArs Editora; 2019.}

José Ignacio Allevi

(https://orcid.org/0000-0003-4819-1398) ${ }^{1}$

${ }^{1}$ Instituto de Humanidades y Ciencias Sociales del Litoral (IHUCSO), Consejo Nacional de Investigaciones Científicas y Técnicas (CONICET). Santa Fe Argentina.

Bien podríamos afirmar que el último libro de Sandra Caponi parte de un acontecimiento: el descubrimiento de la acción psico-neuro-farmacológica de la clorprozamina en 1952. No obstante, su obra reúne más de una historia. Es la pesquisa sobre un fármaco, al tiempo que la historia de una disciplina y sus intentos de cientifización. Es también el retrato de una nueva etapa del capitalismo, de la articulación industrial con el saber médico, y sus implicancias sociales. Pero, además, ofrece una ruta genealógica más para reflexionar sobre la propuesta de subjetivación neoliberal que, ya en 1952, exponía su deriva, hoy tan familiar como amenazante.

Amparado en una concisa revisión bibliográfica y la consulta de archivos específicos en Francia, el libro se estructura en una introducción y seis capítulos, precedidos por presentaciones de Robert Whitacker y Fernando Freitas. Sandra Caponi no podía menos que partir desde el presente para interrogar el pasado. A través de una lectura y uso de la producción de Michel Foucault con ánimos epistemológicos antes que teóricos, la autora retoma el "descubrimiento" de la clorpromazina como hecho inaugural de una nueva etapa en la disciplina psiquiátrica, la de su biologización.

Este último proceso, se enmarca en un trayecto más extenso, pues desde el comienzo del siglo XX la disciplina persiguió diversos mecanismos para aproximarse al campo médico. La malarioterapia, los tratamientos de shock por insulina y cardiazol y las terapias electroconvulsivas hacia finales de los años treinta sustentaron el proceso que aborda el libro de Caponi' ${ }^{1}$. Como señala la autora, la "revolución psicofarmacológica" de 1952 y 1954 dificilmente podría conceptualizarse como instancia superadora bajo un matriz disciplinar kuhniana. En efecto, si bien la clorprozamina modificó radicalmente la posición y alcance de la Psiquiatría en el campo médico y social, una característica estructural permanecía intacta: la Psiquiatría no pudo ni puede especificar su objeto. Tras una miríada de variaciones terminológicas, la locura resulta hoy día etiológicamente inexplicable. Sin embargo, la clorpromazina habilitó nuevas y mayores esferas de intervención a la disciplina, que la autora analiza bajo el prisma de un régimen de verdad foucaultiano, para alcanzar la politicidad que atraviesa el estatuto epistemológico de un saber.

Los primeros tres capítulos del libro son claves para comprender histórica y epistemológicamente el cambio introducido en 1952. En el primero, la autora estudia la dimensión que la psicofarmacología adoptó en la constitución de un nuevo régimen de verdad psiquiátrico. Así, historiza los primeros usos de la clorprozamina como histamínico y anestésico, al tiempo que traza los vínculos que permitieron que sea testeada en los pacientes del Hospital de Saint Anne de Paris, a través de su director, Pierre Deniker.

El segundo capítulo desanda las estrategias por las cuales se persiguió legitimar el uso masivo de esta droga. Aquí, la autora enfatiza lúcidamente la paradoja del proceder psiquiátrico: a diferencia de la medicina, el punto de partida no es una enfermedad, sino la gestión del espacio que contiene a la locura. El modelo naciente de la psicofarmacología, entonces, centró su eje en las drogas antes que en la enfermedad, definiendo los diagnósticos a partir de los efectos de los fármacos. Su "revolución" era doble: no sólo ofrecían una posibilidad concreta para aproximarse al modelo médico. Saldaban también un problema histórico del espacio asilar, al habilitar el tratamiento ambulatorio de la locura. Pero fue con la venta de los laboratorios franceses a capitales norteamericanos cuando la producción y uso de los psicofármacos se tornó desmedida.

La autora explaya con mayor detenimiento la consolidación de la hipótesis dopaminérgica en el tercer capítulo, la cual posibilitó articular diagnósticos e intervenciones dado que los primeros constituían grupos de síntomas, sin etiología concreta. Ante la ausencia de marcadores biológicos, la red causal de la enfermedad se reconstruye sólo a través de la terapéutica. Tras el ingreso de capitales norteamericanos a la producción de psicofármacos, estos procesos epistémicos y tecnológicos señalaron un devenir histórico que vuelve comprensible la sistematización del primer Manual Diagnósticos y Estadístico de Trastornos Mentales (DSM) por la American Psychiatry Association en 1953. Son, entonces, las limitaciones del modelo explicativo de la psiquiatría las que abren posibilidades de investigación -y de nuevos fármacos-ilimitadas. Este cambio de perspectiva desde la enfermedad hacia el fármaco ilumina que los efectos de los mismos responden más a las sustancias que contienen antes que a su probada eficacia terapéutica. Es por ello que el capítulo siguiente se ocupe de explorar tales vínculos. De allí que, en su articulación con una industria en plena expansión durante la segunda posguerra, como la farmacéutica, la "agenda" de dro-

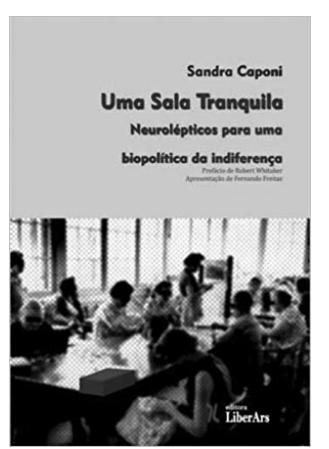


gas que abordasen efectos específicos, o bien que aminorasen contraindicaciones de los primeros, no hiciera más que expandirse.

El quinto capítulo avanza sobre el traspaso entre dispositivos foucautlianos habilitado por las drogas psicotrópicas: de uno disciplinario a otro de seguridad, donde aumenta exponencialmente la lectura psiquiátrica de comportamientos cotidianos. Para observar esta nueva modulación de la gubernamentalidad neoliberal que comenzaba a esbozarse, Caponi opta por un recurso sugerente: estudiar la publicidad de los antipsicóticos y su devenir temporal. Con esta acertada decisión metodológica, la autora sin dudas logró desplazar la lente desde los espacios de producción de saber hacia su circulación masiva. Y, al mismo tiempo, logra exponer con claridad la lógica incesante de producción constante de medicamentos cuya única función era contrarrestar efectos adversos de uno anterior, o bien medicalizar nuevas conductas o sentimientos.

En el último capítulo, la autora recupera una experiencia contemporánea que permite comprender los alcances concretos del proceso iniciado en 1952, sobre la cual pesquisó proficuamente: la sucesiva psiquiatrización de conductas infantiles en las distintas ediciones del DSM, tomando como caso el diagnóstico de TOD (trastorno opositivo desafiante). Con ese propósito, analiza cómo el mismo sustrato epistemológico consolidado a partir de 1952 permitió la creciente expansión taxonómica de cada edición del DSM, y su correlato medicalizante. Al mismo tiempo, se concentra en el uso de un fármaco en particular -la risperidonaen la población infantil, acompañada de nuevos fármacos que mitiguen sus efectos adversos, así como de las preocupantes normativas de salud pública que estimulan su prescripción. Las conclusiones del libro recuperan los aportes centrales y refuerzan el enfoque conceptual de la autora sobre la transformación de la psiquiatría hacia un dispositivo de seguridad, y en especial su propuesta de subjetivación contemporánea.

Pocas dudas caben que Sandra Caponi dio luz a un libro de historia y epistemología de la psiquiatría. No obstante, al analizar las múltiples ramificaciones que tuvo el inusitado descubrimiento de la primera droga psiquiátrica, este trabajo resulta un puntapié ineludible en una historia cultural de la subjetividad contemporánea. Por otra parte, así como la autora afirma que el "último" Foucault registró la politicidad que atraviesa a los saberes que constituyen regímenes de verdad, en igual medida podemos afirmar que este libro es profundamente político. En su afán, esta obra detona lateralmente una serie de interrogantes que enriquecen la reflexión sobre nuestra contemporaneidad. En primer término, cabe revisar el lugar que los Estados de bienestar y sus políticas públicas durante la segunda mitad del siglo XX jugaron en la expansión de prácticas y sentidos hacia una nueva gubernamentalidad, sustentada en valores diametralmente opuestos. En este registro, la expansión de la circulación y consumo de terapias y culturas psi es parte de esta historia ${ }^{2}$. Por otro lado, es posible reflexionar sobre la afinidad electiva que la psiquiatrización o neurologización de las narrativas del yo genera sobre una grilla de inteligibilidad común al neoliberalismo. Dicho en otros términos, cuánto de estas propuestas de subjetividad resulta consonante con la "economización" de las elecciones ${ }^{3}$. Por último, este libro brinda una sólida base histórica y epistemológica para comprender cómo estos saberes, su circulación y sus usos operan en el fenómeno de proliferación de culturas terapéuticas ${ }^{4}$ que apuntalan una gestión del yo contemporáneo donde la "felicidad" se presenta como imperativo categórico 5 .

Frente a un contexto de medicalización agresiva y del rescate constante -explícito o no- de la indiferencia como valor ético, Sandra Caponi nos recuerda, fiel a su adscripción foucaultiana, que la exposición de las raíces históricas de los fenómenos es la mejor manera de evidenciar el absurdo que en ocasiones los sustenta y, con ello, su carácter grotesco.

\section{Referencias}

1. Missa JN. Naissance de la psychiatrie biologique. Historie des traitements des maladies mentales au XXme siécle. Paris: PUF; 2006.

2. Illouz E. La salvación del alma moderna. Terapia, emocionesy cultura dela autoayuda. Buenos Aires: Katz;2010.

3. Foucault M. Nacimiento de la biopolítica. Curso en el Collége de France, 1978-1979. Buenos Aires: FCE; 2007.

4. Papalini V. Culturas terapéuticas: de la uniformidad a la diversidad. Methaodos Rev Cs Soc 2014;2(2):212-226.

5. Cabanas E, Illouz E. Manufacturing happy citizens. How science and industry of happiness control our lives. London: Polity; 2019. 